\title{
Pathways from childhood maltreatment to cardiometabolic disease: a research review
}

Jessie R. Baldwin, $\mathrm{PhD}^{1,2}$ and Andrea Danese, $\mathrm{MD} \mathrm{PhD}^{2,3,4}$

${ }^{1}$ Department of Clinical, Educational and Health Psychology, Division of Psychology and Language Sciences, University College London, London, UK

2Social, Genetic and Developmental Psychiatry Centre, Institute of Psychiatry, Psychology and Neuroscience, King's College London, London, UK

${ }^{3}$ Department of Child and Adolescent Psychiatry, Institute of Psychiatry, Psychology and Neuroscience, King's College London, London, UK

${ }^{4}$ National and Specialist CAMHS Clinic for Trauma, Anxiety, and Depression, South London and Maudsley NHS Foundation Trust, London, UK

Correspondence: Jessie Baldwin, PhD, Department of Clinical, Educational and Health Psychology, Division of Psychology and Language Sciences, University College London, 26 Bedford Way, London, WC1H OAP, UK. Email: j.baldwin@ucl.ac.uk

Word count: 3475

Number of figures: 1

Number of references: 96 


\section{Abstract}

Childhood maltreatment is a risk factor for poor physical health outcomes in later life, such as cardiovascular disease and type 2 diabetes. In order to target preventative interventions to reduce this risk, it is important to understand the biological mechanisms linking childhood maltreatment to cardiometabolic disorders. Research reviewed here suggests that maltreated children show alterations in biological systems relating to metabolism and the immune system, which could in turn increase long-term cardiometabolic disease risk. Specifically, maltreated children show an elevated risk of obesity in adulthood, and high inflammation levels in adolescence and adulthood. These findings from observational studies of humans are consistent with evidence from experimental animal models showing causal effects of early life stress on obesity and immune function. To help prevent maltreated children from developing cardiometabolic disease later in life, clinical assessment and treatment should focus on physical health as well as mental health.

Keywords: childhood maltreatment, childhood victimisation, early life stress, obesity, inflammation, physical health 


\section{PATHWAYS FROM CHILDHOOD MALTREATMENT TO CARDIOMETABOLIC DISEASE:}

A RESEARCH REVIEW

\section{INTRODUCTION}

It is increasingly recognised that maltreated children have an elevated risk of physical health problems later in life. For example, adults with histories of childhood maltreatment show a high prevalence of clinical outcomes, such as cardiovascular disease (Suglia et al., 2018) and type 2 diabetes (Widom et al., 2012). These clinical findings are also supported by experimental animal models showing that early life stress can cause indicators of cardiovascular disease in rodents (Loria et al., 2013) and type 2 diabetes in non-human primates (Kaufman et al., 2007). In order to understand how childhood maltreatment might affect long-term cardiometabolic risk, and to inform preventative strategies, it is important to investigate whether maltreated children develop physical risk factors for later-life disease.

In this paper, we review the literature to describe how exposure to childhood maltreatment could lead to pre-clinical abnormalities in metabolic and immune functioning, which in turn might affect risk for cardiometabolic disease decades after maltreatment has ceased. A growing body of research from observational studies of humans and experimental animal models suggests that childhood maltreatment and other early life stressors can increase risk of obesity and lead to elevated inflammation levels. We will review this research, distinguishing between studies of adults and children to gain insight into the shortand long-term effects of maltreatment. We will also outline potential mechanisms through which maltreatment could exert effects on obesity and inflammation. Finally, we will discuss the clinical implications of this emerging field of research.

\section{CHILDHOOD MALTREATMENT AND OBESITY}

\section{Observational studies in humans}

A number of observational studies in humans have shown that childhood maltreatment is associated with obesity (body mass index $[\mathrm{BMI}] \geq 30 \mathrm{~kg} / \mathrm{m}^{2}$ ) later in life. Indeed, a metaanalysis of 41 studies (including 190,285 participants) showed that childhood maltreatment was associated with a small but significant increase in risk of obesity (odds ratio $=1.36,95 \%$ confidence interval $=1.26-1.47)$ (Danese and Tan, 2014). This association was independent of a number of potential confounders, including socioeconomic status, current smoking, alcohol intake and physical activity. Sensitivity analyses showed that the association between maltreatment and obesity was observed across all maltreatment types, with the exception of emotional neglect. Furthermore, the association was present in adults but not in 
children or adolescents. The latter finding suggested the existence of an 'incubation period' between exposure to childhood maltreatment and development of obesity.

Although inference on an 'incubation period' was initially based on multiple crosssectional studies in the meta-analysis, subsequent findings from longitudinal studies examining developmental trajectories of body mass in maltreated and non-maltreated children were broadly consistent with this hypothesis. For example, one study assessed children exposed to substantiated maltreatment and non-maltreated controls at four timepoints across late childhood and adolescence (Schneiderman et al., 2015). The results showed that females exposed to sexual abuse or neglect had different BMI trajectories than non-maltreated females, in that they showed lower BMI in late childhood/early adolescence and higher BMI in late adolescence. Another study tested the association between childhood maltreatment and BMI measured at seven time-points from childhood to age 50 in participants from the 1958 British birth cohort/National Child Development Study (Power et al., 2015). Participants with reports of physical abuse and neglect (and sexual abuse in females) showed normal BMI in childhood, but faster BMI gain up to age 50 and obesity in adulthood. These associations were independent of parental BMI, prenatal/infancy factors, childhood factors, education, and adult health behaviours. These findings suggest that childhood maltreatment is associated with a progressive increase in risk for obesity over the life-course.

In addition to victimisation by adults in the form of childhood maltreatment, victimisation by peers also appears to be associated with obesity. For example, children exposed to chronic bullying victimisation were more likely to be overweight in young adulthood than non-bullied children (Baldwin et al., 2016). This risk remained even after accounting for genetic risk of overweight, childhood weight, childhood mental health/cognition and other forms of environmental adversity. Furthermore, frequently bullied female children in the 1958 British Birth Cohort had an elevated risk of obesity at mid-life (Takizawa et al., 2015), suggesting that, like childhood maltreatment, bullying victimisation may have long-lasting effects on obesity risk.

\section{Experimental animal models}

Animal models have provided important experimental evidence for a causal effect of early life stress on obesity in later life. Although animal models may be limited in generalisability to human experiences, biology, and behaviour, these experimental studies are uniquely placed to address causal inference because they can manipulate the environment and randomly assign animals to stressful early experiences. This means that pre-existing differences between the animals exposed or not exposed to early life stress can be assumed to be equally distributed between groups, and any differences in obesity between groups can be 
attributed to differential exposure to early life stress. In contrast, because random assignment of human children to maltreatment is clearly unethical, observational human studies rely on statistically controlling for factors that are associated with childhood maltreatment and obesity and may thus act as confounders. However, many of these potential confounding factors are unknown or unmeasured in practice, which limits causal inference in human studies. The complementary strengths of experimental animal models and observational human studies allow confidence to be placed in findings that converge from both methods.

In animals, two studies of non-human primates have suggested that early life stress is causally associated with the development of obesity. First, Kaufman et al. (2007) exposed infant monkeys to early life stress in a paradigm known as variable foraging demand. This involved imposing food insecurity on their mothers for 16 weeks during nursing, which has been shown to impair maternal care and evoke stress responses in the offspring (Andrews and Rosenblum, 1994). The results showed that peripubertal primates who had been exposed to prolonged maternal food insecurity exhibited greater weight, BMI and abdominal circumference than normally reared controls. Second, Conti et al. (2012) carried out a study in which newborn rhesus monkeys were either reared by their mothers (the control condition) or removed from their mothers and reared alongside their peers (the stress condition). In later life, female peer-reared monkeys exhibited greater weight than monkeys reared by their mothers. These experimental findings support evidence from observational human studies showing an association between early life stress and later obesity.

\section{How could childhood maltreatment lead to obesity?}

Childhood maltreatment and other early life stressors could increase obesity risk by giving rise to a 'thrifty' phenotype, characterised by increased energy intake and storage and/or decreased energy expenditure (Danese and Tan, 2014). This could occur via interacting biological and behavioural mechanisms affecting energy intake and expenditure.

Childhood maltreatment and increased energy intake. Maltreated children may eat more food than non-maltreated children due to alterations in reward processing, the neuroendocrine stress system, and executive function. With regard to reward processing, maltreated children show reduced sensitivity to reward, described both at a behavioural level by poorer reward-related task performance (Guyer et al., 2006) and at the neural level by weaker activation of reward-related brain regions in the basal ganglia during reward anticipation (Mehta et al., 2010). In turn, low reward sensitivity could result in maltreated children overeating high-calorie food in order to satisfy a reward-deficit. With regard to the neuroendocrine stress system, maltreated children show chronic overactivation of the central stress response system - the hypothalamic-pituitary-adrenal (HPA) axis (De Bellis et al., 
1999) and associated mental and physical symptoms of anxiety (Tarullo and Gunnar, 2006). Given that high-calorie food can reduce HPA axis activation (Pecoraro et al., 2004) and anxiety (Prasad and Prasad, 1996), maltreated individuals may 'self-medicate' with highcalorie food. With regard to executive function, maltreated children show impulsivity and impairments in inhibitory control (Guyer et al., 2006) which might arise due to smaller volume of the prefrontal cortex, a brain-region involved in these functions (Lim et al., 2014). In turn, poor inhibitory control over food intake could result in maltreated individuals overeating (Wonderlich et al., 2001). These proposed mechanisms are consistent with evidence linking childhood maltreatment to excessive eating (Tarren-Sweeney, 2006) and clinical disorders involving overeating, such as food addiction (Mason et al., 2013) and binge eating disorder (Striegel-Moore et al., 2002).

Childhood maltreatment and decreased energy expenditure. In addition to increased energy intake, maltreated children may have decreased energy expenditure due to alterations in metabolic hormones, mental health problems, and abnormal immune functioning. With regard to metabolic hormones, initial evidence suggests that childhood maltreatment and other early life stressors are associated with low basal and stimulated levels of leptin (Danese et al., 2014; Panagiotaropoulos et al., 2004). Given that leptin promotes lipolysis (the breakdown of fat into energy), leptin deficiency in maltreated children could reduce energy expended through this basal metabolic process. With regard to mental health problems, maltreated children have an elevated risk of developing depression (Widom et al., 2007), which is associated with physical inactivity. Therefore, children exposed to maltreatment might engage in less exercise due to depression. With regard to the immune system, as will be discussed later in this review, maltreated children are at risk of developing high inflammation levels (Danese et al., 2007). Because inflammation can induce fatigue (Dantzer et al., 2008), high inflammation levels could result in maltreated individuals being less physically active. The latter two mechanisms are consistent with evidence showing that adults with a history of childhood maltreatment report engaging in less physical activity than non-maltreated individuals (Power et al., 2015).

Taken together, these findings suggest that childhood maltreatment may increase risk of obesity in adult life via plausible (but largely untested) biological and behavioural mechanisms. In turn, elevated rates of obesity in individuals with a history of childhood maltreatment could at least partly contribute to their increased risk of later cardiometabolic disease.

\section{CHILDHOOD MALTREATMENT AND INFLAMMATION}

\section{Observational studies in humans}


Inflammation is part of the innate immune response to infection and physical injury. Although timely activation of the inflammatory response is a vital defensive mechanism, the inflammatory response is non-specific and can also mistakenly target parts of the body and cause damage when chronically activated. Evidence suggests that childhood maltreatment is associated with chronic low-grade inflammation, as reflected in high levels of inflammation biomarkers such as the acute phase protein C-reactive protein (CRP) and pro-inflammatory cytokines like tumour necrosis factor alpha (TNF- $\alpha$ ) and interleukin 6 (IL-6). For example, children exposed to maternal rejection, harsh discipline, disruptive caregiver changes, physical abuse or sexual abuse showed higher levels of CRP than non-maltreated children at age 32 (Danese et al., 2007). This association also generalised to other inflammation biomarkers such as fibrinogen and white blood cell count. Furthermore, the association was independent of the influence of low birth weight, childhood family socio-economic disadvantage, and low IQ. Since these initial findings, more than two dozen studies have investigated the association between childhood maltreatment and inflammation in adulthood, with qualitative and quantitative reviews showing that adults with histories of childhood maltreatment have a small elevation in CRP, TNF- $\alpha$, and IL-6 (Baumeister et al., 2015; Danese and Baldwin, 2017). These long-term effects of maltreatment have generalised to other forms of early life stress such as bullying victimisation, which also predict high inflammation levels in midlife (Takizawa et al., 2015).

To understand the emergence of the effect of childhood maltreatment on cardiometabolic disease risk, studies have also examined whether maltreated children already showed high inflammation levels in early life. Evidence suggests that childhood maltreatment is associated with a very small elevation in inflammation levels in young people (Kuhlman et al., 2019), though effects can vary according to differing methodological approaches, co-occurring psychopathology, or gender. For example, maltreated children with depression showed elevated CRP levels at age 12, compared to non-maltreated children matched for sex, socioeconomic status, and zygosity (Danese et al., 2011). Furthermore, children exposed to multiple victimisation types (including physical abuse, sexual abuse, emotional abuse and neglect, physical neglect, domestic violence and bullying victimisation) showed elevated levels of CRP at age 18 (Baldwin et al., 2018). Notably, this association was present only in females, and not in males. In females, the association was independent of genetic risk for inflammation, socioeconomic disadvantage, obesity, and body temperature. Consistent with findings in adults, these findings linking childhood maltreatment to inflammation in young people also generalise to other forms of victimisation: for example, children exposed to cumulative bullying victimisation showed elevated CRP levels in adolescence and greater increases in CRP levels from adolescence to young 
adulthood (Copeland et al., 2014).

In addition to an association between childhood maltreatment and elevated baseline inflammation levels, maltreated children also show greater pro-inflammatory responses to subsequent stressors. For example, adults with a history of childhood maltreatment showed a greater inflammatory response to an acute psychosocial stress test than non-maltreated controls (Carpenter et al., 2010). Maltreated individuals also showed heightened inflammatory responses to daily stressors and caregiving stress in older adulthood (Gouin et al., 2012). Furthermore, depressed adults reporting childhood maltreatment showed an elevated inflammatory response to acute stress, relative to controls (Pace et al., 2006).

\section{Experimental animal models}

A number of experimental animal studies have investigated whether early life stress has causal effects on immune function and development. An extensive review of these studies is beyond the scope of this review, but interested readers may refer to Ganguly and Brenhouse (2015). In brief, results vary according to the type of stressor and immune system measure used, though several studies have reported elevated inflammation levels in animals exposed to early maternal separation. For example, Cole et al. (2012) assessed inflammatory gene expression profiles of rhesus monkeys either reared maternally or removed from their mothers and reared with peers. Relative to monkeys reared by their mothers, maternallydeprived monkeys showed enhanced expression of genes involved in inflammation. This suggested that early life stress can increase inflammatory gene expression in the first few months of life in non-human primates. These findings are consistent with experimental findings from rodent studies showing that maternal separation increases inflammatory markers (O'Mahony et al., 2009).

\section{How could childhood maltreatment affect inflammation?}

Maltreated children may develop high inflammation levels via a number of biological and behavioural mechanisms.

Biological mechanisms. At least three potential biological pathways could link childhood maltreatment to inflammation. First, maltreated children show reduced sensitivity to cortisol (a hormone with anti-inflammatory properties), which could in turn result in chronically elevated inflammation levels. Indeed, a longitudinal study found that female adolescents from harsh families showed decreasing sensitivity to cortisol's anti-inflammatory properties and greater inflammatory responses to stressors over time (Miller and Chen, 2010). Similarly, adolescents exposed to early life stress showed resistance to cortisol and increased levels of inflammation biomarkers (do Prado et al., 2017). Second, childhood 
maltreatment is associated with elevated risk of infections, which could impact immune development. For example, maltreated children are more likely to be exposed to infections and injury (Gilbert et al., 2009) and may also be more susceptible to infection (Shirtcliff et al., 2009). In turn, exposure to multiple infections could result in chronic inflammation. Third, childhood maltreatment could increase inflammation levels due to alterations in the microbiome. For example, early life stress in animals can cause alterations in the colonisation and composition of the gut microbiota (O'Mahony et al., 2009). In turn, alterations in the gut microbiome during sensitive periods in early life could affect immune system development and lead to chronic inflammation (Cryan and Dinan, 2012).

Behavioural mechanisms. As well as biological pathways between childhood maltreatment and inflammation, several broader behavioural mechanisms may operate. First, disruption of sleep patterns could partly mediate the association between childhood maltreatment and high inflammation levels. Maltreated children have an elevated risk of sleep problems (Cecil et al., 2015), and experimental studies have shown that sleep deprivation can cause increased inflammation levels (Meier-Ewert et al., 2004). Second, obesity is likely to play a role in the association between childhood maltreatment and inflammation. As discussed, maltreated children have an elevated risk of obesity (Danese and Tan, 2014), and obesity is linked to a chronic inflammatory state due to production of pro-inflammatory cytokines by adipose (fat) tissue (Gregor and Hotamisligil, 2011). Notably, one study showed that the relationship between childhood maltreatment and CRP levels in females was mediated through obesity (Matthews et al., 2014), though other studies found that the association was independent of obesity (Danese et al., 2011; Danese et al., 2007; Baldwin et al., 2018). Third, maltreated children may develop high inflammation levels due to consuming addictive substances. Adults with histories of childhood maltreatment are particularly likely to smoke and abuse alcohol and drugs (Felitti et al., 1998), which can increase inflammation levels . These addictive behaviours may therefore partly explain the high inflammation levels in adults exposed to childhood maltreatment (Danese and Baldwin, 2017).

\section{DISCUSSION}

Maltreated children show a higher risk of developing obesity and higher inflammation levels than non-maltreated children. These effects appear to progressively emerge throughout adolescence and become established by adulthood (Power et al., 2015; Baumeister et al., 2015). Because these metabolic and immune abnormalities after exposure to early life stress in humans appear to be conserved across other species with distant common ancestors (e.g., non-human primates), it is possible that excess weight and inflammation may be adaptive in the face of early life stress (Danese and Baldwin, 2017). For example, larger body weight could help abused children to better defend or protect themselves against 
violence and sexual victimisation. In addition, high levels of body fat could help ensure that neglected children have adequate energy supply during periods of malnutrition. Furthermore, high inflammation levels could accelerate wound repair and prevent infections in children with injuries from violence and infection due to unsanitary environments.

Despite these potential adaptive advantages of obesity and inflammation in the context of childhood maltreatment, when maltreated individuals escape abusive and neglectful environments and priorities shift from survival to long-term health, obesity and inflammation will become maladaptive by conferring disease risk (Danese and Baldwin, 2017). Both obesity and inflammation are major independent risk factors for cardiovascular disease (Ridker et al., 1997) and type 2 diabetes (Reilly and Kelly, 2011). Because these clinical outcomes are more prevalent in adults with histories of childhood maltreatment, it is possible that obesity and high inflammation might help explain why childhood maltreatment is associated with increased disease risk in later life. Though future research is required to test whether obesity and inflammation mediate the effects of childhood maltreatment on later disease risk, the current findings reviewed here have clinical implications.

\section{Clinical implications}

Given that maltreated children are at higher risk for physical health problems as well as mental health problems, professionals working with maltreated children need to be aware of the potential for poor physical health and integrate its management in their practice. To detect risk of poor physical health in maltreated children, professionals should adopt more holistic approaches to assessment. For example, maltreated children could be assessed for BMI, blood pressure, heart rate, immune-related conditions (e.g., asthma, allergies) and routine blood tests, as well as mental health and psychosocial needs. Notably, looked-after children do undergo regular physical health screenings, and the findings above suggest that physical health screening would benefit all maltreated children regardless of care arrangements. Professionals and carers are in the position to put in place interventions to prevent maltreated children from developing physical health problems. It is still unclear which interventions may be effective in preventing physical health outcomes among maltreated children. However, we have summarised the hypothesised pathways from childhood maltreatment to adult clinical outcomes in Figure 1, and these pathways suggest potential targets for interventions. Building on some of the targets identified in Figure 1, professionals and carers could support maltreated children in engaging in healthy behaviours, such as eating sensibly, exercising, and abstaining from smoking, alcohol and drugs, which can have a substantial impact on physical health. Given that health behaviours during early life track into adulthood, establishing healthy habits in maltreated children could reduce their future risk of physical health problems and potentially also improve mental health outcomes. 
Furthermore, professionals and carers have an important role in promoting mental health, and emerging evidence suggests that treatments for mental health problems can lower preclinical physical health risks in maltreated children (Pace et al., 2013; Bower and Irwin, 2016).

\section{Conclusion}

Research reviewed here suggests that childhood maltreatment may confer long-term risk to cardiometabolic disease through biological pathways involving abnormalities in metabolism and the immune system (Suglia et al., 2018). Specifically, children exposed to maltreatment show a heightened risk of obesity and an elevation in inflammation levels, which are apparent in adulthood but may start emerging earlier in adolescence. To prevent maltreated children from becoming obese and developing high inflammation levels, professionals working with maltreated children should regularly assess their physical health and help them to establish long-term healthy habits. Future research should also further characterise the mechanisms through which maltreated children develop risk of obesity and high inflammation levels, so that targeted interventions can be developed to reduce maltreated children's long-term disease risk.

Figure 1. Hypothesised pathways from childhood maltreatment to adult clinical outcomes.

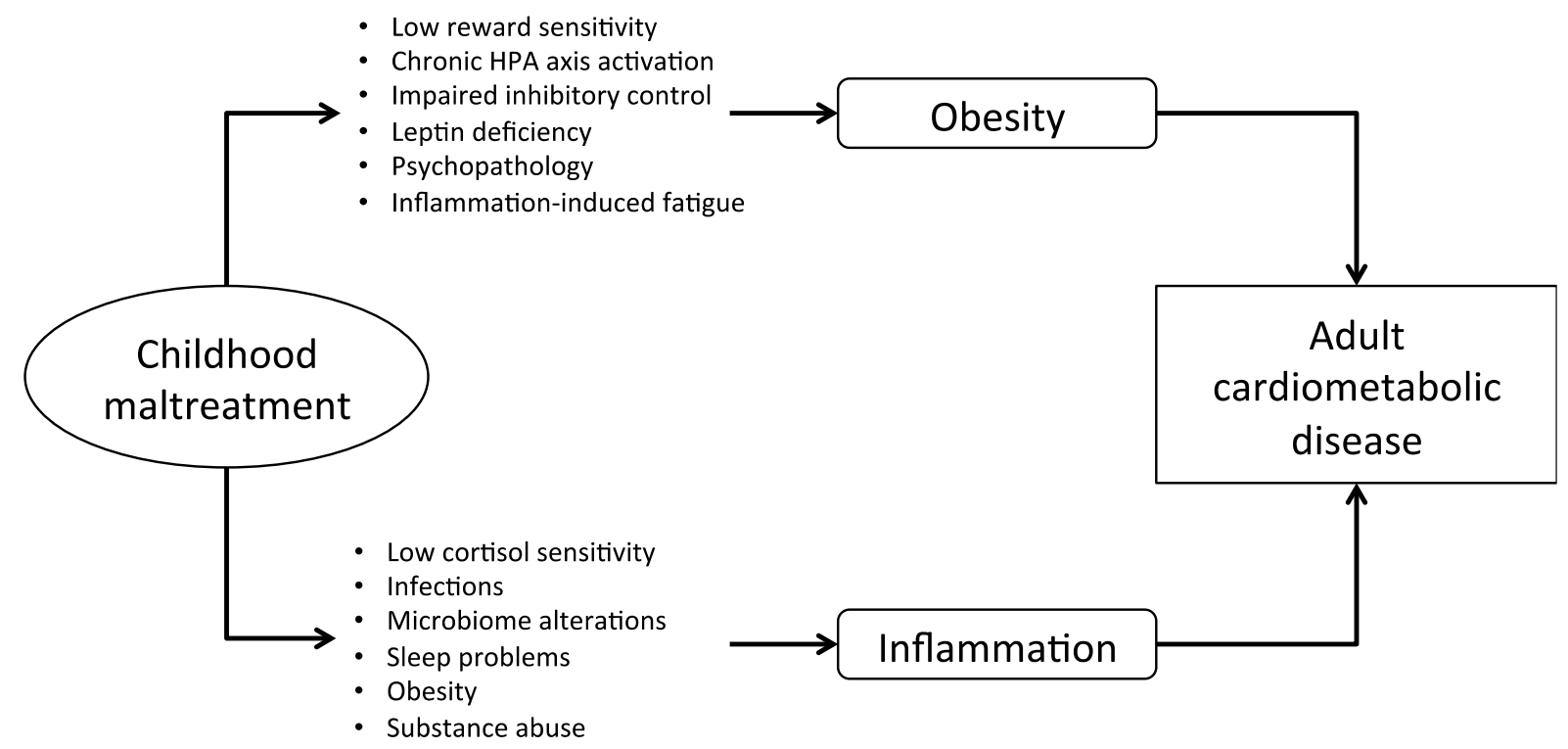




\section{References}

Andrews MW and Rosenblum LA. (1994) The development of affiliative and agonistic social patterns in differentially reared monkeys. Child Development 65: 1398-1404.

Baldwin JR, Arseneault L, Caspi A, et al. (2018) Childhood victimization and inflammation in young adulthood: A genetically sensitive cohort study. Brain, Behavior, and Immunity 67: 211-217.

Baldwin JR, Arseneault L, Odgers C, et al. (2016) Childhood bullying victimization and overweight in young adulthood: A cohort study. Psychosomatic Medicine 78: 10941103.

Baumeister D, Akhtar R, Ciufolini S, et al. (2015) Childhood trauma and adulthood inflammation: a meta-analysis of peripheral C-reactive protein, interleukin-6 and tumour necrosis factor- $\alpha$. Molecular Psychiatry 21: 642-649.

Bower JE and Irwin MR. (2016) Mind-body therapies and control of inflammatory biology: a descriptive review. Brain, Behavior, and Immunity 51: 1-11.

Carpenter LL, Gawuga CE, Tyrka AR, et al. (2010) Association between plasma IL-6 response to acute stress and early-life adversity in healthy adults. Neuropsychopharmacology 35: 2617-2623.

Cecil CA, Viding E, McCrory EJ, et al. (2015) Distinct mechanisms underlie associations between forms of childhood maltreatment and disruptive nocturnal behaviors. Developmental Neuropsychology 40: 181-199.

Cole SW, Conti G, Arevalo JM, et al. (2012) Transcriptional modulation of the developing immune system by early life social adversity. Proceedings of the National Academy of Sciences 109: 20578-20583.

Conti G, Hansman C, Heckman JJ, et al. (2012) Primate evidence on the late health effects of early-life adversity. Proceedings of the National Academy of Sciences 109: 88668871.

Copeland WE, Wolke D, Lereya ST, et al. (2014) Childhood bullying involvement predicts low-grade systemic inflammation into adulthood. Proceedings of the National Academy of Sciences 111: 7570-7575.

Cryan JF and Dinan TG. (2012) Mind-altering microorganisms: the impact of the gut microbiota on brain and behaviour. Nature Reviews Neuroscience 13: 701-712.

Danese A and Baldwin JR. (2017) Hidden wounds? Inflammatory links between childhood trauma and psychopathology. Annual Review of Psychology 68: 517-544.

Danese A, Caspi A, Williams B, et al. (2011) Biological embedding of stress through inflammation processes in childhood. Molecular Psychiatry 16: 244. 
Danese A, Dove R, Belsky DW, et al. (2014) Leptin deficiency in maltreated children. Translational Psychiatry 4: e446.

Danese A, Pariante CM, Caspi A, et al. (2007) Childhood maltreatment predicts adult inflammation in a life-course study. Proceedings of the National Academy of Sciences 104: 1319-1324.

Danese A and Tan M. (2014) Childhood maltreatment and obesity: systematic review and meta-analysis. Molecular Psychiatry 19: 544-554.

Dantzer R, O'Connor JC, Freund GG, et al. (2008) From inflammation to sickness and depression: when the immune system subjugates the brain. Nature Reviews Neuroscience 9: 46-56.

De Bellis MD, Baum AS, Birmaher B, et al. (1999) Developmental traumatology part I: Biological stress systems. Biological Psychiatry 45: 1259-1270.

do Prado CH, Grassi-Oliveira R, Daruy-Filho L, et al. (2017) Evidence for immune activation and resistance to glucocorticoids following childhood maltreatment in adolescents without psychopathology. Neuropsychopharmacology 42: 2272-2282.

Felitti VJ, Anda RF, Nordenberg D, et al. (1998) Relationship of childhood abuse and household dysfunction to many of the leading causes of death in adults. American Journal of Preventive Medicine 14: 245-258.

Ganguly P and Brenhouse HC. (2015) Broken or maladaptive? Altered trajectories in neuroinflammation and behavior after early life adversity. Developmental Cognitive Neuroscience 11: 18-30.

Gilbert R, Kemp A, Thoburn J, et al. (2009) Recognising and responding to child maltreatment. The Lancet 373: 167-180.

Gouin J-P, Glaser R, Malarkey WB, et al. (2012) Childhood abuse and inflammatory responses to daily stressors. Annals of Behavioral Medicine 44: 287-292.

Gregor MF and Hotamisligil GS. (2011) Inflammatory mechanisms in obesity. Annual Review of Immunology 29: 415-445.

Guyer AE, Kaufman J, Hodgdon HB, et al. (2006) Behavioral alterations in reward system function: the role of childhood maltreatment and psychopathology. Journal of the American Academy of Child and Adolescent Psychiatry 45: 1059-1067.

Kaufman D, Banerji MA, Shorman I, et al. (2007) Early-life stress and the development of obesity and insulin resistance in juvenile bonnet macaques. Diabetes 56: 1382-1386.

Kuhlman KR, Horn SR, Chiang JJ, et al. (2019) Early life adversity exposure and circulating markers of inflammation in children and adolescents: A systematic review and metaanalysis. Brain, Behavior, and Immunity.

Lim L, Radua J and Rubia K. (2014) Gray matter abnormalities in childhood maltreatment: a voxel-wise meta-analysis. American Journal of Psychiatry 171: 854-863. 
Loria AS, Brands MW, Pollock DM, et al. (2013) Early life stress sensitizes the renal and systemic sympathetic system in rats. American Journal of Physiology-Renal Physiology 305: F390-F395.

Mason SM, Flint AJ, Field AE, et al. (2013) Abuse victimization in childhood or adolescence and risk of food addiction in adult women. Obesity 21: E775-E781.

Matthews KA, Chang Y-F, Thurston RC, et al. (2014) Child abuse is related to inflammation in mid-life women: role of obesity. Brain, Behavior, and Immunity 36: 29-34.

Mehta MA, Gore-Langton E, Golembo N, et al. (2010) Hyporesponsive reward anticipation in the basal ganglia following severe institutional deprivation early in life. Journal of Cognitive Neuroscience 22: 2316-2325.

Meier-Ewert HK, Ridker PM, Rifai N, et al. (2004) Effect of sleep loss on C-reactive protein, an inflammatory marker of cardiovascular risk. Journal of the American College of Cardiology 43: 678-683.

Miller GE and Chen E. (2010) Harsh family climate in early life presages the emergence of a proinflammatory phenotype in adolescence. Psychological Science 21: 848-856.

O'Mahony SM, Marchesi JR, Scully P, et al. (2009) Early life stress alters behavior, immunity, and microbiota in rats: implications for irritable bowel syndrome and psychiatric illnesses. Biological Psychiatry 65: 263-267.

Pace TW, Mletzko TC, Alagbe O, et al. (2006) Increased stress-induced inflammatory responses in male patients with major depression and increased early life stress. American Journal of Psychiatry 163: 1630-1633.

Pace TW, Negi LT, Dodson-Lavelle B, et al. (2013) Engagement with cognitively-based compassion training is associated with reduced salivary C-reactive protein from before to after training in foster care program adolescents.

Psychoneuroendocrinology 38: 294-299.

Panagiotaropoulos T, Papaioannou A, Pondiki S, et al. (2004) Effect of neonatal handling and sex on basal and chronic stress-induced corticosterone and leptin secretion. Neuroendocrinology 79: 109-118.

Pecoraro N, Reyes F, Gomez F, et al. (2004) Chronic stress promotes palatable feeding, which reduces signs of stress: feedforward and feedback effects of chronic stress. Endocrinology 145: 3754-3762.

Power C, Pereira SMP and Li L. (2015) Childhood maltreatment and BMI trajectories to midadult life: follow-up to age 50y in a British birth cohort. PloS One 10: e0119985.

Prasad A and Prasad C. (1996) Short-term consumption of a diet rich in fat decreases anxiety response in adult male rats. Physiology and Behavior 60: 1039-1042. 
Reilly JJ and Kelly J. (2011) Long-term impact of overweight and obesity in childhood and adolescence on morbidity and premature mortality in adulthood: systematic review. International Journal of Obesity 35: 891-898.

Ridker PM, Cushman M, Stampfer MJ, et al. (1997) Inflammation, aspirin, and the risk of cardiovascular disease in apparently healthy men. New England Journal of Medicine 336: 973-979.

Schneiderman J, Negriff S, Peckins M, et al. (2015) Body mass index trajectory throughout adolescence: a comparison of maltreated adolescents by maltreatment type to a community sample. Pediatric Obesity 10: 296-304.

Shirtcliff EA, Coe CL and Pollak SD. (2009) Early childhood stress is associated with elevated antibody levels to herpes simplex virus type 1. Proceedings of the National Academy of Sciences 106: 2963-2967.

Striegel-Moore RH, Dohm F-A, Pike KM, et al. (2002) Abuse, bullying, and discrimination as risk factors for binge eating disorder. American Journal of Psychiatry 159: 19021907.

Suglia SF, Koenen KC, Boynton-Jarrett R, et al. (2018) Childhood and adolescent adversity and cardiometabolic outcomes: a scientific statement from the American Heart Association. Circulation 137: e15-e28.

Takizawa R, Danese A, Maughan B, et al. (2015) Bullying victimization in childhood predicts inflammation and obesity at mid-life: a five-decade birth cohort study. Psychological Medicine 45: 1-11.

Tarren-Sweeney M. (2006) Patterns of aberrant eating among pre-adolescent children in foster care. Journal of Abnormal Child Psychology 34: 621-632.

Tarullo AR and Gunnar MR. (2006) Child maltreatment and the developing HPA axis. Hormones and Behavior 50: 632-639.

Widom CS, Czaja SJ, Bentley T, et al. (2012) A prospective investigation of physical health outcomes in abused and neglected children: New findings from a 30-year follow-up. American Journal of Public Health 102: 1135-1144.

Widom CS, DuMont K and Czaja SJ. (2007) A prospective investigation of major depressive disorder and comorbidity in abused and neglected children grown up. Archives of General Psychiatry 64: 49-56.

Wonderlich S, Crosby R, Mitchell J, et al. (2001) Pathways mediating sexual abuse and eating disturbance in children. International Journal of Eating Disorders 29: 270-279. 